\title{
Draft Genome Sequences of Elsinoë fawcettii and Elsinoë australis Causing Scab Diseases on Citrus
}

\author{
Gnanendra Shanmugam, ${ }^{1}$ Junhyun Jeon, ${ }^{1,2, \dagger}$ and Jae-Wook Hyun ${ }^{3, \dagger}$ \\ ${ }^{1}$ Department of Biotechnology, Yeungnam University, Gyeongsan, Gyeongbuk, 38541, Korea \\ ${ }^{2}$ Plant Immunity Research Center, Seoul National University, Seoul, 08826, Korea \\ ${ }^{3}$ Citrus Research Institute, National Institute of Horticultural and Herbal Science, Rural Development \\ Administration, Jeju 63607, Korea
}

\begin{abstract}
Elsinoë fawcettii and E. australis (phylum Ascomycota) are phytopathogenic fungi causing scab diseases on citrus plants. We report here the high-quality draft genome sequences and ab initio gene predictions of two $E$. fawcettii strains and one $E$. australis strain, which differ in their host range. This genome sequence information will provide valuable resources to underpin genomic attributes for determining host range through comparative genomic analyses of citrus scab fungi.
\end{abstract}

Citrus scab disease is one of the most prevalent fungal diseases in many citrusproducing areas (Kunta et al. 2013). The disease affects not only the leaves and twigs but also fruit of susceptible cultivars causing fruit blemishes, which greatly reduce the value of fruit for the fresh market (Chung 2011). Two species of fungi are generally recognized as causal agents of scab diseases: Elsinoë fawcettii and $E$. australis, causing citrus scab (sour orange scab or common scab) (Gopal et al. 2014) and sweet orange scab (Chung 2011), respectively. Both fungal species belong to the phylum Ascomycota, class Dothideomycetes, order Myriangiales, and family Elsinoaceae. The symptoms of citrus scab vary depending on citrus cultivars and timing of infection but they often appear as warty pustules that are raised on leaves and fruit. There are mainly six pathotypes of $E$. fawcettii: Florida broad host range (FBHR), Florida narrow host range, Tryon's, Lemon, Jingeul, and SRGC; and two pathotypes in E. australis: sweet orange and natsudaidai (Hyun et al. 2009). Both E. fawcettii and E. australis are known to produce elsinochrome, a nonhost-selective perylenequinonecontaining phytotoxin, a prerequisite for full fungal virulence and lesion formation (Chung 2011).

E. fawcettii SM16-1 was isolated from satsuma mandarin (SM) in Jeju Island, Korea and E. fawcettii DAR70024 from Citrus indica in New South Wales, Australia. E. australis Ea-1 was obtained from Argentina. E. fawcettii SM16-1 and DAR70024 and E. australis Ea-1 were classified into different pathotypes according to their ability to infect differential cultivars (E. fawcettii SM16-1, FBHR; E. fawcettii DAR70024, Tryon's; and E. australis Ea-1, sweet orange) (Hyun et al. 2009). Based on the phylogenetic tree obtained from the alignment of internal transcribed spacer and translation elongation factor sequences, isolates were identified as $E$. fawcettii and $E$. australis.

The genome sequencing of three isolates was performed with Illumina technology using paired-end and mate-pair libraries (GreenGene BioTech, Yong-In, Korea). In total, 403,757,008 paired-end and mate-pair raw reads of $101 \mathrm{bp}$ were generated for $E$. fawcettii SM16-1, while

${ }^{\dagger}$ Corresponding authors: J. Jeon; jjeon@yu.ac.kr; and J.-W. Hyun; hyunjaewook@korea.kr

The author(s) declare no conflict of interest.

Accepted for publication 1 October 2019.

(C) 2020 The American Phytopathological Society
Funding

This work was supported by a grant (PJ013178) from the Rural Development Administration, Republic of Korea.

Keywords

genome, citrus scab, Elsinoë 
$399,958,543$ reads for $E$. fawcettii DAR70024 and 379,895,328 reads for $E$. australis Ea-1 were generated. FASTQC was used to check the quality of the reads. The low-quality bases with Phred score less than Q33 and adapter sequence contamination were trimmed by using TrimGalore-0.5.0 and Cutadpat-2.1 to obtain $385,060,456$ reads, which corresponds to approximately $128 \times$ sequence depth. De novo assemblies of all three genomes reported in this study was performed by using SPAdes genome assembler 3.9.0 (Bankevich et al. 2012) with default settings. The de novo assembly of $E$. fawcettii SM16-1 reads resulted in a genome size of $26,655,928 \mathrm{bp}, \mathrm{G}+\mathrm{C}$ content of $52.79 \%$, and 1,276 contigs with a scaffold length at which $50 \%$ of the total assembly length is covered $\left(\mathrm{N}_{50}\right)$ value of $952.195 \mathrm{bp}$ and the number of contigs whose summed length is $N_{50}\left(L_{50}\right)$ of 6. SSPACE-3.0 (Boetzer et al. 2011) was used for scaffolding to obtain the final version, with 72 total scaffolds and a total genome size of 26.65 $\mathrm{Mb}$. The de novo assembly of two other isolates, E. fawcettii DAR70024 (26.32 Mb) and E. australis Ea-1 (23.79 Mb), produced 1,650 contigs with $\mathrm{N}_{50}$ value of 843,756 bp and 1,750 contigs with $\mathrm{N}_{50}$ value of $620,754 \mathrm{bp}$, respectively. In comparison, the genome of $E$. australis $N L 1$, available in the NCBI public database (accession NHZQ00000000), is $23.22 \mathrm{Mb}$ with $52.7 \%$ GC content. E. australis NL1 genome consists of 453 scaffolds with $N_{50}$ value of 897,805 , and $L_{50}$ of 9 ; in total, 9,223 genes were reported on this genome (Table 1).

The ab initio gene prediction using Augustus (Keller et al. 2011) (-species = fusarium graminearum -strand $=$ both -gene model = complete) for the assembled genome of E. fawcettii SM16-1 resulted in 10,340 (exons $=27,304$ and introns $=17,063$ ) proteincoding genes, while 9,930 (exons $=26,910$ and introns $=16,991$ ) and 9,002 (exons = 24,384 and introns $=15,387$ ) protein-coding genes were predicted on $E$. fawcettii DAR70024 and E. australis Ea-1, respectively. The genome assembly completeness for E. fawcettii SM16-1 (82\%), E. fawcettii DAR70024 (92\%), and E. australis Ea-1 (91\%) was determined by BUSCO v3 (Waterhouse et al. 2018), using the fungi dataset.

The functional annotation of genes was done by searching homology of predicted genes with Ascomycetes protein sequences from SwissProt (Bairoch and Apweiler 2000) using BLASTP (Altschul et al. 1990). The functional annotation of $E$. fawcettii SM16-1 (10,340 genes), E. fawcettii DAR70024 (9,930 genes), and E. australis Ea-1 (9002 genes) revealed that $83.87 \%(8,673), 82.81 \%(8,224)$, and $86.54 \%(7,791)$ of genes were annotated, respectively. The pathogenicity genes were predicted by using BLASTP against the Pathogen-Host Interaction (PHI) database (Winnenburg et al. 2006), which identified 981 $(9.48 \%), 963(9.69 \%)$, and $873(9.69 \%)$ putative PHI genes in E. fawcettii SM16-1, E. fawcettii DAR70024, and E. australis Ea-1, respectively. Secreted proteins were predicted by first identifying proteins harboring signal peptides using SignalP 3.0 (Nielsen 2017) and then removing the proteins with mitochondria and chloroplast targeting signals using TargetP (Emanuelsson et al. 2007). This revealed that 382 (3.69\%), 367 (3.69\%), and 328 (3.64\%) proteins are putative secreted proteins in E. fawcettii SM16-1, E. fawcettii DAR70024, and E. australis Ea-1, respectively.

Carbohydrate-active enzymes (CAZymes) that play a vital role in breakdown of cell wall components of the host to establish a successful infection process were predicted by using CAT and dbCAN servers (Yin et al. 2012). The CAZymes analysis revealed that 3,205 (30.99\%), 2,979 (30\%), and 2,790 (30.99\%) proteins from E. fawcettii SM16-1, E. fawcettii

Table 1. Genome assembly statistics of assembled Elsinoë fawcettii strains and E. australis

\begin{tabular}{lccc} 
& \multicolumn{3}{c}{ E. fawcettii } \\
\cline { 2 - 3 } & SM16-1 & DAR70024 & E. australis Ea-1 \\
Pathotype & FBHR $^{\mathrm{a}}$ & Sweet orange & Tryon's \\
NCBI accession & VAAB00000000 & SWCR00000000 & SWCS00000000 \\
Genome size (Mb) & 26.65 & 26.32 & 23.79 \\
Contigs & 1,276 & 1,650 & 1,750 \\
$\mathrm{~N}_{50}$ (bp) & 952,195 & 843,756 & 620,754 \\
GC content (\%) & 52.79 & 52.29 & 52.64 \\
Number of genes & 10,340 & 9,930 & 9,002 \\
Average gene length (bp) & 1,621 & 1,693 & 1,718 \\
Number of annotated genes & 8,673 & 8,224 & 7,791 \\
Number of unannotated genes & 1,667 & 1,706 & 1,211 \\
\hline
\end{tabular}

a $\mathrm{FBHR}=$ Florida broad host range. 
DAR70024, and E. australis Ea-1, respectively, are CAZymes. Further classification of CAZymes based on their catalytic activity showed that glycosyl hydrolases $(1,162$ [36.25\%], 1,027 [34.47\%], and 1,046 [37.49]) are high in number, followed by glycoside transferases (1,057 [32.88\%], 978 [32.82\%], and 981 [35.16\%]), carbohydrate esterases (387 [12.07\%], 406 [13.62\%], and 307 [11\%]), carbohydrate-binding modules (271 [8.45\%], 257 [8.62\%], and 194 [6.95\%]), auxiliary activities (317 [9.89\%], 292 [9.80\%], and 251 [8.99\%]), and polysaccharide lyases (14 [0.42\%], 19 [0.63\%], and 11 [0.39\%]) in E. fawcettiiSM16-1, E. fawcettii DAR70024, and E. australis Ea-1, respectively.

Secondary metabolites are essential for fungal growth and development, providing protection against various environmental stresses (Calvo et al. 2002). The search for secondary metabolite clusters on the E. fawcettii SM16-1, E. fawcettii DAR70024, and E. australis Ea-1 genome using the anti-SMASH fungal version (Blin et al. 2017) revealed the presence of 23 clusters (8 NRPS-like, 6 T1PKS, 5 NRPS, 3 terpene, and 1 lanthipeptide), 21 clusters (8 NRPS-like, 6 T1PKS, 4 NRPS, and 3 terpene), and 20 clusters (7 NRPS-like, 5 NRPS, 4 T1PKS, and 4 terpene), respectively. Elsinochrome, a photosensitizing compound, is reported to be highly toxic to citrus by causing peroxidation of cell membranes and inducing rapid electrolyte leakage from tissues (Chung 2011). The secondary metabolite cluster analysis showed the presence of T1PKS encoding elsinochrome A ( $E$. fawcettii SM16-1) and elsinochrome B/C (E. fawcettii DAR70024 and E. australis Ea-1) cluster.

The draft genomes of E. fawcettii SM16-1, E. fawcettii DAR70024, and E. australis Ea-1 reported in this study are of high-quality genome assemblies, which can serve as reference genomes for other species or strains within the family Elsinoaceae. The genomes of E. fawcettii SM16-1, E. fawcettii DAR70024, and E. australis Ea-1 reported here have been deposited at GenBank under the accession numbers VAAB00000000 (BioProject number PRJNA534191), SWCR00000000 (BioProject number PRJNA534185), and SWCS00000000 (BioProject number PRJNA534188), respectively.

\section{Literature Cited}

Altschul, S. F., Gish, W., Miller, W., Myers, E. W., and Lipman, D. J. 1990. Basic local alignment search tool. J. Mol. Biol. 215:403-410.

Bairoch, A., and Apweiler, R. 2000. The SWISS-PROT protein sequence database and its supplement TrEMBL in 2000. Nucleic Acids Res. 28:45-48.

Bankevich, A., Nurk, S., Antipov, D., Gurevich, A. A., Dvorkin, M., Kulikov, A. S., Lesin, V. M., Nikolenko, S. I., Pham, S., Prjibelski, A. D., Pyshkin, A. V., Sirotkin, A. V., Vyahhi, N., Tesler, G., Alekseyev, M. A., and Pevzner, P. A. 2012. SPAdes: A new genome assembly algorithm and its applications to single-cell sequencing. J. Comput. Biol. 19:455-477.

Blin, K., Wolf, T., Chevrette, M. G., Lu, X., Schwalen, C. J., Kautsar, S. A., Suarez Duran, H. G., de Los Santos, E. L. C., Kim, H. U., Nave, M., Dickschat, J. S., Mitchell, D. A., Shelest, E., Breitling, R., Takano, E., Lee, S. Y., Weber, T., and Medema, M. H. 2017. antiSMASH 4.0-Improvements in chemistry prediction and gene cluster boundary identification. Nucleic Acids Res. 45:W36-W41.

Boetzer, M., Henkel, C. V., Jansen, H. J., Butler, D., and Pirovano, W. 2011. Scaffolding pre-assembled contigs using SSPACE. Bioinformatics 27: 578-579.

Calvo, A. M., Wilson, R. A., Bok, J. W., and Keller, N. P. 2002. Relationship between secondary metabolism and fungal development. Microbiol. Mol. Biol. Rev. 66: 447-459.

Chung, K. R. 2011. Elsinoë fawcettii and Elsinoë australis: The fungal pathogens causing citrus scab. Mol. Plant Pathol. 12:123-135.

Emanuelsson, O., Brunak, S., von Heijne, G., and Nielsen, H. 2007. Locating proteins in the cell using TargetP, SignalP and related tools. Nat. Protoc. 2: 953-971.
Gopal, K., Govindarajulu, B., Ramana, K. T. V., Kishore Kumar, Ch S., Gopi, V., Gouri Sankar, T., Mukunda Lakshmi, L., Naga Lakshmi, T., and Sarada, G. 2014. Citrus scab (Elsinoë fawcettii): A review. Res. Rev. J. Agric. Allied Sci. 3:49-58.

Hyun, J. W., Yi, S. H., Mackenzie, S. J., Timmer, L. W., Kim, K. S., Kang, S. K., Kwon, H. M., and Lim, H. C. 2009. Pathotypes and genetic relationship of worldwide collections of Elsinoë spp. causing scab diseases of citrus. Phytopathology 99: 721-728.

Keller, O., Kollmar, M., Stanke, M., and Waack, S. 2011. A novel hybrid gene prediction method employing protein multiple sequence alignments. Bioinformatics 27:757-763.

Kunta, M., Rascoe, J., de Sa, P. B., Timmer, L. W., Palm, M. E., da Graça, J. V., Mangan, R. L., Malik, N. S. A., Salas, B., Satpute, A., Sétamou, M., and Skaria, M. 2013. Sweet orange scab with a new scab disease "syndrome" of citrus in the USA associated with Elsinoë australis. Trop. Plant Pathol. 38:203-212.

Nielsen, H. 2017. Predicting secretory proteins with SignalP. Methods Mol. Biol. 1611:59-73.

Waterhouse, R. M., Seppey, M., Simão, F. A., Manni, M., loannidis, P., Klioutchnikov, G., Kriventseva, E. V., and Zdobnov, E. M. 2018. BUSCO applications from quality assessments to gene prediction and phylogenomics. Mol. Biol. Evol. 35: 543-548.

Winnenburg, R., Baldwin, T. K., Urban, M., Rawlings, C., Köhler, J., and HammondKosack, K. E. 2006. PHI-base: A new database for pathogen host interactions. Nucleic Acids Res. 34:D459-D464.

Yin, Y., Mao, X., Yang, J., Chen, X., Mao, F., and Xu, Y. 2012. dbCAN: A web resource for automated carbohydrate-active enzyme annotation. Nucleic Acids Res. 40:W445-W451. 